\title{
Caracterización de prácticas de cuidado en familias colombianas con niños en primera infancia en situación de desplazamiento forzado*
}

Characteristics of Caregiving Practices in Colombian Families with Children in Early Childhood in Forcibly Displaced Situation

\author{
Olga Alicia María Carbonell Blanco*** \\ SANDRA Juliana Plata CONTRERAS \\ Milton EdUARDo Bermúdez JaIMES \\ LILIANA CATALINA SuÁREZ BaraCALDo \\ Paola Andrea Peña Patiño \\ Carolina Villanueva Betancourth \\ Pontificia Universidad Javeriana, Bogotá, Colombia
}

doi: 10.11144/Javeriana.upsy14-1.cpcf

Para citar este artículo: Carbonell, O. A. M., Plata, S. J., Bermúdez, M. E., Suárez, L. C., Peña, P. A., \& Villanueva, C. (2015). Caracterización de prácticas de cuidado en familias colombianas con niños en primera infancia en situación de desplazamiento forzado. Universitas Psychologica, 14(1), 67-80. http://dx.doi.org/10.11144/Javeriana.upsy14-1.cpcf

* Artículo original resultado de investigación

** Facultad de Psicología. Correos electrónicos: carbonel@javeriana.edu.co, sandra.plata@javeriana. edu.co,milton.bermudez@javeriana.edu.co,catalinita_22@hotmail.com,paolaplus@hotmail.com, carolinavillanuevab@gmail.com
RESUMEN

El propósito del estudio fue caracterizar las prácticas de crianza del cuidador principal respecto a calidad del cuidado, prácticas disciplinarias, relaciones de apego tempranas niño-cuidador y desarrollo infantil en 10 diadas caso (cuidador-hijo), en situación de desplazamiento forzado por conflicto armado interno en Colombia con niños en primera infancia. El diseño fue mixto explicativo con instrumentos observacionales derivados de la Teoría del Apego y medidas de autorreporte. Se encontraron asociaciones significativas entre sensibilidad del cuidador con desarrollo infantil $(r=$ $0.63, p<0.05)$, entre apego infantil con dominios de cuidado materno $(r$ $=0.91, p<0.01)$ y prácticas disciplinarias moderadas asociadas negativamente con la seguridad del apego infantil $(r=-0.88, p<0.01)$. Siguiendo los principios metodológicos de la Teoría Fundamentada, se obtuvieron categorías emergentes sobre transmisión intergeneracional de patrones de crianza y prácticas de cuidado infantil. Los resultados permiten identificar algunos factores protectores (procesos reflexivos respecto al uso de patrones inadecuados de crianza) y de riesgo (mantenimiento de prácticas de castigo físico, en especial con niñas).

Palabras clave

prácticas de crianza; apego; sensibilidad del cuidador; conflicto armado;

desplazamiento forzado

\section{A B S T R A C T}

The study's main purpose was to characterize the quality of caregiving, disciplinary practices, child attachment security, and child development in 10 families' study cases with their young children at internal armed conflict and forcibly displaced. The methodological design was a mixed methods explanatory design. Data collected with instruments derived from Attachment Theory, and self-report measures showed significant associations between caregiver sensitivity and child development $(r=0.63, p<0.05)$, and between child attachment security and maternal caregiving domains $(r=0.91, p<0.01)$. Non-harsh disciplinary practices are significant and negatively associated to child attachment security $(r=-0.88, p<0.01)$. Following methodological principles based on Grounded Theory emergent categories were obtained about intergenerational transmission of childrearing practices and current parental caregiving practices. Findings identified 
some protective factors (reflective processes regarding inadequate childrearing patterns) and risk factors (maintenance of physical punishment practices, especially with girls).

Keywords

childrearing practices; attachment; caregiver sensitivity; armed conflict; forcibly displaced

\section{Introducción}

Durante más de 40 años en Colombia se han recrudecido los enfrentamientos armados entre diferentes actores del conflicto. Estos enfrentamientos han forzado a millones de personas y a sus familias a dejar sus tierras, por amenazas a su integridad física y psicológica, y a desplazarse a los centros suburbanos y urbanos a lo largo y ancho del país. Bogotá está siendo una de las ciudades con mayor recepción de esta población (Amnistía Internacional, 2009; Consultoría para los Derechos Humanos y el Desplazamiento [CODHES], 2009).

En este escenario de conflicto armado, la primera infancia ha sido quizás la más vulnerable y expuesta a situaciones violentas, siendo testigos directos e indirectos del conflicto (Fondo de las Naciones Unidas para la Infancia [Unicef], 1996, 2014). Lo que conlleva a que sean los niños y las niñas las principales víctimas, teniendo impactos en la seguridad física, estrés postraumático y secuelas en el desarrollo emocional, social, cognitivo y afectivo (Connolly, Hayden, Levin, \& Shulman, 2009).

A pesar que Unicef (2006) señala la importancia de la familia como entorno protector durante y luego del conflicto armado, los padres se sienten profundamente afectados y traumatizados por las vivencias experimentadas, y en esa medida la calidad del cuidado de sus hijos se ve afectada. El sistema comportamental de cuidado se refiere a proveer al niño cuidados físicos, y especialmente dar protección en situaciones de riesgo o peligro a través de conductas de proximidad y contacto (Bowlby, 1993). La calidad de cuidado de acuerdo a Ainsworth, Bell y Stayton (1974) ha sido conceptualizada y evaluada partir de cuatro indicadores comportamentales que se hallan en un continuo, tales como la cooperación-interferencia con las conductas de autonomía del niño, la disponibilidad física y psicológica del cuidador-ignorar/ negligencia con las señales y necesidades del niño, la aceptación-rechazo del cuidador y por último, la sensibilidad-insensibilidad a las señales y comunicaciones del niño.

Desde la teoría del apego para entender la calidad del cuidado, es necesario referirse al modelo de la transmisión intergeneracional de las relaciones de apego y de los patrones de crianza como el proceso mediante el cual, de forma intencionada o no, una generación anterior influye psicológicamente en las actitudes y el comportamiento de los padres de la siguiente generación (Berlin, 2007; Berlin, Zeanah, \& Lieberman, 2008).

Diversos estudios han mostrado que existe una asociación significativa entre la calidad de cuidado materno y la seguridad del apego infantil (metaanálisis de De Wolff \& van IJzendoorn, 1997; Posada, Carbonell, Álzate, \& Plata, 2004). No obstante, se ha encontrado, con algunas excepciones, que en las muestras de sectores más pobres de la población la asociación entre ambas variables es más baja, lo cual parece estar relacionado con eventos estresantes del contexto familiar y de las condiciones de vida (De Wolff \& van IJzendoorn, 1997).

Por otra parte, la crianza es un proceso que implica asumir una responsabilidad a través de una serie de acciones e interacciones por parte de los cuidadores, para promover el desarrollo emocional, social y físico de los niños con particularidades culturales (Myers-Walls, Myers-Bowman, \& Posada, 2005). No obstante, las exigencias del ambiente o situaciones persistentes como la pobreza, producen estrés en la crianza, que tiene un impacto adverso y reduce la capacidad de la familia para afrontar las demandas parentales, lo cual afecta negativamente la calidad de las relaciones y el cuidado materno (Wachs, 2009), a su vez asociado con la seguridad del apego de los niños (Hadadian \& Mebler, 1996 citados por Mulsow, Caldera, Pursley, Reifman, \& Huston 2002).

La literatura ha mostrado también que en diversos contextos de adversidad psicosocial los niños muestran mayor probabilidad de desarrollar relaciones de apego inseguras (apego desorganizado) espe- 
cialmente cuando los padres transmiten patrones de cuidado con maltrato y negligencia, pues se afecta la confianza básica en los adultos cuidadores presentes y futuros (Gómez Muzzio, Muñoz, \& Santelices, 2008). Así mismo, los niños en ambiente de pobreza tienen menor acceso a actividades cognitivamente estimulantes (Bornstein \& Putnick, 2012), lo cual afecta su neurodesarrollo, así como su desempeño en tareas cognitivas de lenguaje, memoria, memoria de trabajo y control cognitivo (Farah et al., 2006).

Sin embargo, las familias en condiciones de vulnerabilidad tienen un potencial de resiliencia, la cual es definida como un proceso con factores protectores que predicen resultados favorables y una adaptación positiva en el desarrollo de los niños y sus familias, a pesar de estar expuestos a contextos de bastante adversidad (Kim-Cohen, Moffitt, Caspi, \& Taylor, 2004). Los dos componentes básicos de la resiliencia son la exposición al riesgo y la evidencia de una buena adaptación de la persona, entendida no como una característica individual, sino como el resultado de un proceso contextual, relacional y familiar (Delage, 2010; Egeland, Carlson, \& Sroufe, 1993). Entre los factores protectores en estos contextos de adversidad, como el conflicto armado, los estudios han mostrado que los cuidadores que comparten tiempo con los niños y tienen cercanía emocional con ellos, así como contar con apegos estables y seguros al interior de las familias, favorece los procesos resilientes en la familia (Wyman et al., 1999).

Desde una perspectiva ecológica-transaccional del desarrollo (Sagi-Schwartz, 2012), es posible apreciar influencias reciprocas protectoras y/o de riesgo entre los sistemas en los cuales está inmerso el niño expuesto al conflicto armado. El sistema intraindividual del niño podría potencialmente verse afectado a nivel neurológico por el estrés severo, así como su capacidad de regulación emocional, comportamental y social; sin embargo, las personas cercanas del microsistema familiar pueden filtrar estas experiencias adversas y revertir los efectos negativos del primer sistema, proveyendo un ambiente protector, relaciones de confianza y un refugio de seguridad para el niño. Es decir, que una buena competencia parental puede moderar el impacto negativo de las experiencias traumáticas y se vuelve en un factor de resiliencia. De la misma manera, en el exosistema, la comunidad, sitio de trabajo de los padres, la escuela también pueden convertirse en refugios de seguridad y apoyo para los padres, lo cual a su vez se revierte en capacidad para proteger a los hijos, e igualmente, el desarrollo del niño puede ver influenciado positiva o negativamente y de manera indirecta por creencias, valores, actitudes en el macrosistema.

La problemática del desplazamiento forzado ha tenido múltiples estudios que consideran factores macro y mesosociales; sin embargo, son pocas las investigaciones realizadas en el país respecto a factores microsociales, que hayan indagado las problemáticas familiares y relaciones en la vida cotidiana de estas familias (Arango, Acosta, \& Jaramillo, 2009; González Viveros, 2004). Realizar estudios que hagan caracterizaciones de las relaciones familiares y de la crianza infantil en estos contextos permite reconocer las fortalezas, las fragilidades y los recursos con los que cuentan estas familias para afrontar de una manera resiliente la crianza de sus hijos.

En consecuencia, el presente estudio caracterizó la calidad del cuidado, las relaciones de apego tempranas niño-cuidador principal, las prácticas de crianza en la dimensión del manejo de las normas y límites y el desarrollo infantil en contextos familiares con niños en primera infancia, expuestos a situaciones de desplazamiento forzado; y así mismo, buscó establecer algunos factores protectores y de riesgo que inciden en las relaciones de crianza y cuidado en estas familias, desde una doble perspectiva: la mirada de observadores externos y la del cuidador participante.

\section{Método}

\section{Diseño}

El diseño metodológico del estudio fue mixto-explicativo, con el fin de caracterizar mejor el fenómeno de las prácticas de cuidado y de crianza de niños en primera infancia en un contexto familiar de riesgo. Se utilizaron estudios de caso para documentar y comprender con mayor profundidad estas prácticas, 
sin pretensiones de generalización de los hallazgos (Hernández Sampieri, Fernández Collado, \& Baptista Lucio, 2010).

\section{Participantes}

Utilizando un muestreo no probabilístico, se seleccionaron 10 familias caso, en situación de desplazamiento forzado con hijos entre 30-66 meses de edad $(M=47.7 ; D E=11.26), 6$ niñas y 4 niños, reubicados en la ciudad de Bogotá. Los cuidadores principales fueron 9 madres y un padre. La edad de los cuidadores estaba en un rango entre 21-30 años con $M=25.3$ y $D E=2.83$; el nivel educativo se encontraba en un rango entre 2 a 16 años de educación con $M=8.5$ años y $D E=4$. El estatus marital de las familias estaba compuesto por $70 \%$ en unión libre, $20 \%$ solteros y $10 \%$ casados. El número de hijos por familia estaba distribuido en 3 hijos (40\%), 2 hijos (30\%) y 1 hijo (30\%). Las familias eran pertenecientes a los estratos socioeconómicos 2 (80 \%) y 3 (20\%), sobre una escala de 6 (Uribe-Mallarino, 2008).

\section{Instrumentos}

\section{Calidad del cuidado}

Fue observada con el Q-sort del Comportamiento Materno para niños menores de tres años. ([MBQS], Pederson \& Moran, 1995), el Q-sort del Comportamiento Materno para Preescolares ([MBPQS], Posada, Jacobs, \& Richmond, 1998) y las Escalas Cualitativas del Comportamiento Materno (Álzate, Carbonell, Posada, \& Bustamante, 1999). El MBQS consta de 90 ítems basados en las conceptualizaciones de Ainsworth et al. (1974) sobre el constructo de sensibilidad del cuidador. La validación del MBQS ha sido reportada por (Pederson, Gleason, Moran, \& Bento, 1998) y la validación del MBPQS por Posada, Kaloustian, Richmond, \& Moreno (2007). El MBPQS está basado en trabajo teórico y empírico sobre relaciones de apego en la infancia y niñez temprana, entrevistas y observaciones de madres de niños preescolares (Ainsworth et al., 1974, 1978; Bowlby,
1993; Cicchetti, Cummings, Greenberg, \& Marvin, 1990; Pederson \& Moran, 1995). En este estudio se obtuvo una confiabilidad interobservadores $(\mathrm{M}=$ 0.86, $\mathrm{DE}=0.05$, Rango $=0.78$ a 0.93).

Las Escalas Cualitativas del Comportamiento Materno son un instrumento que consta de $11 \mathrm{sub-}$ escalas: 1. Prontitud de la respuesta, 2. Efectividad de la respuesta, 3. Consistencia comportamental, 4. Equilibrio entre las demandas del bebé y otras demandas, 5. Equilibrio entre el cuidado físico y la interacción social y emocional con el bebé, 6 . Disfrute de la interacción, 7. Fluidez interactiva, 8. Frecuencia del contacto físico, 9. Calidad del contacto físico, 10. Frecuencia de las verbalizaciones y 11. Diversidad de las funciones en las comunicaciones verbales maternas (Álzate et al., 1999). Estas Escalas mostraron validez concurrente con el MBQS (Pederson \& Moran, 1995; Posada et al., 2004). En este estudio se halló una confiabilidad interobservadores promedio $(\mathrm{M}=0.84, \mathrm{DE}=0.12$, Rango $=0.73$ a 1.0 ).

\section{Las relaciones de apego con el cuidador principal}

La organización del comportamiento de base segura de los niños fue descrita con el Q-sort del Apego ([AQS]; Waters, 1995). Se obtuvo una confiabilidad interobservadores promedio $(\mathrm{M}=0.81, \mathrm{DE}=$ 0.08 , Rango $=0.66$ a 0.91).

\section{Prácticas de crianza}

Fueron evaluadas a través de tres instrumentos: la Encuesta del Comportamiento Familiar-Parte 4 (Posada \& Waters, 1990), el Inventario de Paternidad para Adultos y Adolescentes ([IPAA], Solís-Cámara \& Bavolek, 1996) y una entrevista semiestructurada (Strauss \& Corbin, 2002). El primer instrumento consta de 52 ítems, con una escala Likert de 5 puntos y evalúa la frecuencia en el uso de prácticas disciplinarias, durante los 6 últimos meses. A través de análisis factorial exploratorio se identificaron dos subescalas: prácticas disciplinarias severas y prácticas disciplinarias moderadas. El segundo instrumento posee cuatro subescalas: 1 . Expectativas 
Inapropiadas del Desarrollo, 2. Falta de Empatía, 3. Castigo Corporal y 4. Inversión de Rol Padre-Hijo.

En población mexicana todas las subescalas poseen una confiabilidad interna de 0.77 (Solís-Cámara \& Díaz, 2006). En este estudio, únicamente las subescalas de Castigo Corporal e Inversión de Rol Padre-Hijo mostraron una consistencia interna adecuada (alpha de Cronbach $=0.76$ y 0.79 , respectivamente). El tercer instrumento, la entrevista etnográfica semiestructurada, se diseñó para explorar a profundidad los dominios del cuidado y la crianza en la población específica. Se hizo una validación de contenido por parte de tres jueces expertos en la temática del desplazamiento forzado y en metodologías cualitativas.

\section{Desarrollo infantil}

Se utilizó la Escala Abreviada de Desarrollo (EAD1), para hacer una valoración global de cuatro áreas del desarrollo: motricidad gruesa, motricidad finoadaptativa, audición-lenguaje y personal-social, con un análisis de confiabilidad que oscila entre 0.956 y 0.992 (Ortiz, 1991).

\section{Procedimiento}

Cada familia fue visitada en dos ocasiones en su casa con una duración de 2.5 horas aproximadamente. En la primera visita domiciliaria, se firmó el consentimiento informado avalado por el Comité de Ética e Investigaciones de la Facultad de Psicología de la Pontificia Universidad Javeriana y se aplicó una encuesta sociodemográfica al cuidador principal. Dos observadores, de manera independiente, se encargaron de observar el comportamiento del niño durante las interacciones y rutinas de cuidado diario y un tercer observador se focalizó en el comportamiento del cuidador. Igualmente, se evaluó el desarrollo del niño con apoyo del cuidador principal y finalmente se grabó el audio de una entrevista abierta al cuidador principal para explorar las prácticas de cuidado. En la segunda visita domiciliaria (con un promedio de distancia de visitas de 11 días), se aplicaron dos cuestionarios al cuidador principal, para caracterizar las prácticas de crianza. Por parte de dos observadores independientes se registró la calidad de las interacciones hijo-cuidador principal, y un tercer observador se centró en la observación del comportamiento del niño, durante las interacciones. En ambas visitas domiciliarias un observador filmaba las interacciones cuidador principal-niño.

\section{Plan de análisis de los datos}

Por ser un diseño mixto explicativo de acuerdo con los criterios de Creswell y Plano-Clark (2011), primero se procesaron los datos cuantitativos provenientes de los Q-sorts, los cuestionarios y la escala del desarrollo infantil, con pruebas de estadística descriptiva. Seguidamente, los datos cualitativos que emergieron de la entrevista fueron analizados utilizando las técnicas y estrategias de la Teoría Fundamentada (Strauss \& Corbin, 2002). En la parte final, se procedió a realizar la combinación de los datos cuantitativos con los análisis cualitativos.

Para la interpretación de los resultados, se comparó y se trianguló la información de las observaciones sistemáticas, de los cuestionarios y la entrevista. Como parte de los análisis interpretativos del estudio, se plantearon unas hipótesis preliminares hacia la construcción de una teoría emergente resultante de la indagación cualitativa. La información cualitativa permitió ampliar la comprensión de vacíos y posibles inconsistencias que los datos cuantitativos arrojaron. Al comparar e integrar la información recolectada a través de ambos métodos, fue posible comprender algunos factores de riesgo y protectores que inciden en las relaciones de crianza y cuidado en estas familias.

\section{Resultados}

En la Tabla 1 se presentan correlaciones de Pearson que caracterizan variables sociodemográficas y de cuidado materno.

En la Tabla 1, se observa que a mayor edad del niño más sensible es el comportamiento del cuidador a sus señales comunicativas y necesidades. Igualmente, las madres de mayor edad de la muestra muestran en sus interacciones menor frecuencia 


\section{TABLA 1}

Asociaciones entre variables sociodemográficas y variables de cuidado: sensibilidad del cuidador y subescalas del comportamiento materno

\begin{tabular}{|c|c|c|c|c|c|}
\hline Variable & 1 & 2 & 3 & 4 & 5 \\
\hline 1. Edad del niño & -. & -0.025 & $0.71^{*}$ & -0.16 & 0.1 \\
\hline 2. Edad del cuidador principal & & -. & -0.45 & $0.68^{*}$ & $-0.73 *$ \\
\hline 3. Sensibilidad del cuidador & & & -. & -0.3 & -0.1 \\
\hline 4. Subescala comportamiento Materno-Frecuencia contacto Físico & & & & -. & -0.37 \\
\hline 5. Subescala Comportamiento Materno-Frecuencia comunicativa Verbal & & & & & -. \\
\hline
\end{tabular}

$*_{p}<0.05$. ** $p<0.01$ bilateral.

Fuente: elaboración propia

TABLA 2

Pruebas-t por sexo del niño en prácticas disciplinarias severas y moderadas

\begin{tabular}{|c|c|c|c|c|c|}
\hline Variable & $N$ & M & $D E$ & $t$ & $g l$ \\
\hline \multicolumn{6}{|c|}{ Prácticas Disciplinarias Severas } \\
\hline Niña & 6 & 28 & 24.02 & $2775^{*}$ & 8 \\
\hline Niño & 4 & 0.75 & 0.96 & & \\
\hline \multicolumn{6}{|c|}{ Prácticas Disciplinarias Moderadas } \\
\hline Niña & 6 & 40.83 & 24.7 & $2338 *$ & 8 \\
\hline Niño & 4 & 10.5 & 7.72 & & \\
\hline
\end{tabular}

${ }^{*} p<0.05 . * * p<0.01$ bilateral.

Fuente: elaboración propia

TABLA 3

Asociaciones entre las variables sensibilidad del cuidador, apego, desarrollo infantil, escalas de motricidad gruesa, audición y lenguaje, escalas de castigo corporal e inversión de roles

\begin{tabular}{lccccccc}
\hline Variables & 1 & 2 & 3 & 4 & 5 & 6 & 7 \\
\hline 1.Sensibilidad del cuidador & - & $0.63^{*}$ & $0.69^{*}$ & $0.65^{*}$ & 0.08 & 0.13 & 0.48 \\
2.Desarrrollo Infantil & & - & $0.92^{* *}$ & $0.98^{* *}$ & -0.09 & 0.49 & 0.62 \\
3. Motricidad Gruesa & & & -- & $0.88^{* *}$ & 0.14 & 0.54 & $0.69^{*}$ \\
4.Audición y Lenguaje & & & & - & -0.14 & 0.55 & 0.6 \\
5. Inversión de Roles & & & & & -- & 0.27 & $0.64^{*}$ \\
6. Apego agregado & & & & & &.- & 0.63 \\
7. Castigo Corporal & & & & & &.- \\
\hline
\end{tabular}

$* p<0.05$. * $\mathrm{p}<0.01$ bilateral.

Fuente: elaboración propia

comunicativa verbal y mayor frecuencia de contacto físico.

La Tabla 2 muestra el uso de prácticas disciplinarias severas y moderadas consideradas por sexo, se observa que existen diferencias significativas entre los puntajes obtenidos por niños y niñas. Las medias de los puntajes en ambas escalas son más altas en las niñas.

En la Tabla 3, se observan asociaciones significativas y positivas de correlaciones Pearson entre la sensibilidad del cuidador con el desarrollo infantil, motricidad gruesa y audición y lenguaje. 


\section{TABLA 4}

Asociaciones entre las variables apego infantil, escalas del comportamiento materno y prácticas disciplinarias moderadas

\begin{tabular}{|c|c|c|c|c|c|c|c|c|c|c|}
\hline Variables & 1 & 2 & 3 & 4 & 5 & 6 & 7 & 8 & 9 & 10 \\
\hline Apego visita 1 & -. & $0.67^{*}$ & $0.67 *$ & $0.68^{*}$ & $0.70 *$ & $0.85^{*}$ & -0.06 & $0.89 * *$ & $0.97 * *$ & $-0.88 * *$ \\
\hline $\begin{array}{l}\text { Puntaje total escalas comportamiento } \\
\text { Materno }\end{array}$ & & - & $0.91 * *$ & $0.94 * *$ & $0.88 * *$ & $0.70 *$ & -0.047 & 0.46 & 0.58 & $-0.68 *$ \\
\hline $\begin{array}{l}\text { Subescala Efectividad Respuesta } \\
\text { materna }\end{array}$ & & & -. & $0.85^{* *}$ & $0.85 * *$ & 0.57 & -0.069 & 0.56 & 0.63 & -0.61 \\
\hline $\begin{array}{l}\text { Subescala Consistencia Respuesta } \\
\text { materna }\end{array}$ & & & & -. & $0.89 * *$ & 0.60 & -0.16 & 0.54 & 0.63 & -0.56 \\
\hline $\begin{array}{l}\text { Subescala Fluidez interactiva } \\
\text { materna }\end{array}$ & & & & & - & 0.62 & -0.30 & 0.46 & 0.59 & -0.57 \\
\hline $\begin{array}{l}\text { Subescala Calidad contacto físico } \\
\text { materno }\end{array}$ & & & & & & - & 0.63 & $0.71 *$ & $0.82 * *$ & $-0.68 *$ \\
\hline $\begin{array}{l}\text { Subescala Frecuencia Comunicativa } \\
\text { verbal }\end{array}$ & & & & & & & - & -0.20 & -0.15 & 0.34 \\
\hline Apego visita 2 & & & & & & & & -. & $-0.97 * *$ & $-0.94 * *$ \\
\hline Apego agregado visitas 1 y 2 & & & & & & & & & -. & $0.93^{* *}$ \\
\hline Prácticas disciplinarias moderadas & & & & & & & & & & -. \\
\hline
\end{tabular}

$* p<0.05 * * p<0.01$ bilateral

Fuente: elaboración propia

También se encontró una asociación significativa y positiva entre castigo físico y motricidad gruesa, así como entre las escalas de inversión de roles y castigo físico.

En la Tabla 4, se observan asociaciones entre el apego infantil observado en la visita 1 , con los dominios de cuidado materno: efectividad de la respuesta materna, fluidez interactiva y calidad del contacto físico materno. Así mismo, las prácticas disciplinarias moderadas están inversamente asociadas al apego infantil, al puntaje total de las escalas del comportamiento materno y específicamente a la subescala de calidad del contacto físico.

\section{Análisis cualitativo}

Para la realización del análisis intersujetos de la entrevista cualitativa, se tuvo en cuenta cómo caracterizan los cuidadores desde su propio discurso las prácticas de crianza. A continuación se presentan varias macrocategorías emergentes con sus respectivas subcategorías, utilizando el procedimiento de análisis (codificación abierta -denominación de categorías y subcategorías-, codificación axial, codificación selectiva, matrices intrasujeto e intersujetos) (Strauss \& Corbin, 2002).
Primera macrocategoría: Comportamientos del cuidador principal cuando el niño se porta mal. Con dos subcategorías: principales formas de castigo y acciones diferentes al castigo. $\mathrm{Al}$ indagarse por la subcategoría principales formas de castigo usadas por los cuidadores, se encontró que utilizan como uno de los castigos más frecuentes el quitar privilegios, como juguetes, televisión, salidas o lo que más les guste; "No, pues quitarle las cosas que (...) por un tiempo las cosas que a ellos más le gustan (...) lo que a ellos más les encanta hacer, de pronto podría ser no dejarlos ver televisión, no dejarlos jugar, creo yo que es eso más que todo" (D1111). Sin embargo, dentro de esta subcategoría existen voces débiles que expresan dos formas particulares y quizá preocupantes de quitar privilegios, que van en contra de las necesidades básicas del ser humano, tales como la alimentación y el afecto. La madre D1106 dentro su narración expuso que una forma de dictar normas y limites es quitarle a su hijo la opción de comer un alimento que es de su agrado: "-No te doy de comer, porque es que no tengo, de donde voy a sacar?. Y yo lo castigué, yo no le di arepas esa noche - y se acuesta así y mañana ya que amanezca bien ya le doy, pero yo esa noche lo acosté así y todo y con todo que no aprendió" (D1106). La madre 
D1104 le dice a su hija que "no la vuelve a consentir", si ella repite un comportamiento inadecuado.

En cuanto a la segunda subcategoría acciones diferentes frente al castigo, aparecen otras formas de manejo ante el mal comportamiento de los niños, tales una actitud comprensiva, de reflexión y diálogo, distinta a cualquier forma maltratante por parte del cuidador: "reflexionar como se debería comportar" (D1108) y "tratar de entenderlo, hablar con el niño y explicándole porque no lo puede hacer" (D1107).

La segunda macrocategoría emergente es "la percepción parental de su propia crianza y educación", con tres subcategorías: tipo de trato recibido, reflexión sobre trato recibido y cuidarlo diferente para no repetir la historia. En primer lugar, al indagar por las percepciones que tienen los padres acerca de cómo fueron educados y criados cuando niños, se encontró que la primera subcategoría el tipo de trato que ellos recibieron, este se basó principalmente en un maltrato físico violento y con uso de objetos; por ejemplo, diferentes familias expusieron sus experiencias: "mi mamá me dio también a mi mucho, mucho rejo" (D1101), "mi papá me daba palo por donde me entrara, por nada" (D1108) y "castigo con palo y correa" (D1102). De igual forma, se identificaron otras prácticas, como por ejemplo, castigar con la elaboración de trabajo doméstico y con la falta de afecto por parte de la madre.

Sin embargo, es relevante mostrar que los padres reflexionaron acerca de la manera como fueron tratados. Por ejemplo, D1108 mencionó que: "porque si mi papá fue tremendo conmigo, yo no, no quiero ser igual con mis hijos"; así mismo, D1109 expresó: "porque si nosotros quedamos pues muy dolidos de que nos maltrataron, a ellos no hay que maltratarlos porque ellos van a sentir los que nosotros sentimos" y D1107 señaló que "no se deberían cometer los mismo errores con los hijos". Por ende, los padres reconocen que esta forma si influye en la forma como ellos tratan a sus hijos, pues como mencionó D1103 "Es difícil olvidar y utilizar otra forma para corregir a los hijos".

Al comparar esta subcategoría con las principales formas de castigo que usan los padres con sus hijos, se observó que los padres establecen diferen- cias entre la forma como ellos fueron criados en su infancia y la manera como ellos crían a sus niños en la actualidad, lo cual fue reconocido por la mayoría de participantes.

Dos casos llamaron la atención, respecto a la repetición de patrones de crianza: el cuidador D1110 mencionó que anteriormente castigaba a los hijos de la misma forma en que fue criado, y D1106 reconoce usar el castigo físico así como lo hicieron con ella y no por eso sentir odio hacia las personas que la criaron. También se pueden observar algunas voces débiles acerca de la forma como algunos participantes fueron criados y las percepciones que estos tienen sobre dicho hecho. Por ejemplo, los cuidadores D1101 y D1106 justificaron el tipo de trato recibido en su infancia diciendo que esto había sucedió porque: "su mamá quería el bien para ella" (D1101) o "porque lo hacían para corregirla" (D1106). Por otro lado, la participante D1103 reconoce que el tipo de trato recibido le generó una serie consecuencias psicológicas, como por ejemplo haber sentido que la gente le dice: "que ella es amargada y antisocial", o recordar siempre sus vivencias cuando castiga a su hijo; por esta razón, expone que ella usa una serie de estrategias para no repetir lo mismo con su hijo y con su familia, tales como: "Pedirle a Dios ayuda para entender cómo se sienten sus hijos cuando les pega" y "la mamá debe calmarse, para hablar después y solucionar un problema" (D1103).

A través de sus macrocategorías y subcategorías emergentes, esta entrevista permitió lograr una mayor profundización para comprender las conductas y percepciones que los cuidadores principales tienen sobre las prácticas de crianza y su rol de cuidador. Al referirse a estas y al conectarlas con su propia experiencia de ser cuidados por la generación anterior y al trato recibido por sus padres, ocho cuidadores reportaron que fueron niños maltratados física, verbal y psicológicamente, solo una madre experimentó falta de atención y cuidado psicológico y otra madre manifestó que no fue maltratada.

Así mismo, la subcategoría emergente reflexión sobre el trato recibido, mostró que la mayoría de los padres habían realizado un proceso reflexivo personal sobre las experiencias vividas en su propia infancia y manifestaban que no las querían repetir 
con sus propios hijos. A través de la subcategoría 3 quieren cuidar a sus hijos de manera diferente a como ellos fueron cuidados y tratados, ellos muestran que desean romper el ciclo intergeneracional del maltrato infantil. Sin embargo, narrativas de los cuidadores ya expuestas, permiten suponer que, a pesar del proceso reflexivo, la mayoría de los padres ha llevado a cabo respecto a su propia experiencia de crianza y la aparición de formas diversas de imponer normas y límites a sus hijos, los padres parecen requerir estrategias novedosas para usar una disciplina sensible que no sea coercitiva, que implique reforzamiento positivo, negociación, así como entendimiento y empatía por el niño (Juffer, Barkermans-Kranenburg, \& van IJzendoorn, 2008) y de esa manera poder aprender a co-regular las comportamientos inadecuados de sus hijos y no volver a repetir el patrón de crianza violento.

\section{Análisis integrativo de datos cuantitativos y cualitativos: a manera de discusión}

Los resultados de las escalas de prácticas disciplinarias moderadas y severas, que fueron evaluadas con la Encuesta del Comportamiento Familiar-Parte 4, se corroboran con los hallazgos cualitativos de la entrevista etnográfica semiestructurada de prácticas de crianza y rol de cuidado, respecto a la subcategoría principales formas de castigo, en cuanto a que el castigo físico ha disminuido como práctica disciplinaria principal, si se compara con el patrón comportamental disciplinario utilizado por los padres de la generación anterior y solo se usa como último recurso, cuando los padres consideran que han agotado todas sus formas disciplinarias disponibles, generándoles sentimientos ambivalentes. Es decir, castigo físico impulsivo, producido por sentimientos de enojo, frustración y falta de control (Thompson Gershoff, 2002). No obstante, las prácticas disciplinarias severas, aunque disminuidas, se ejercen más con las niñas. Los hallazgos de este estudio se contradicen con la mayoría de los estudios que muestran que las madres y padres tienden a utilizar mayor castigo físico con los niños que con las niñas, debido a expectativas respecto a la crianza basadas en género, tales como que los niños deben ser educados con mayor dureza para hacerlos más fuertes (Thompson Gershoff, 2002). Sin embargo, los datos de medicina forense en Colombia (Velasco Bernal \& Lozano Mancera, 2012) muestran un crecimiento ascendente de la violencia contra las mujeres a partir de los 5 años de edad.

Así mismo, la información reportada por los padres con el IPAA, en la escala de castigo físico, corrobora los hallazgos de los dos instrumentos anteriores, en esta escala seis cuidadores principales obtienen puntajes que corresponden a decatipos altos a partir de los puntajes brutos obtenidos, lo que implica que creen posible utilizar estrategias disciplinarias alternativas al castigo físico. Cuatro cuidadores principales se ubican en un decatipo norma, de acuerdo al puntaje bruto obtenido, que involucra la creencia de usar el castigo como una de las estrategias disciplinarias además de otras. Esto es coherente con los hallazgos que muestran que las prácticas disciplinarias moderadas se presentan con mayor frecuencia que las severas, como fue reportado por los cuidadores en los dos instrumentos de autorreporte, donde ellos informan sobre otras formas de castigo como: retiro de privilegios (quitar juguetes, no dar dulces, no permitir ver televisión), castigo verbal y psicológico (regaños, hablar fuerte e ignorar al niño-no accesibilidad), aislamiento (encerramiento) y amenazas de pegar y de contarle al papá, especialmente con las niñas.

Por otra parte, los cuidadores también reportan otras acciones para manejar el comportamiento inadecuado de los niños, basadas en el razonamiento (diálogo y explicación del mal comportamiento), lo cual permite suponer que han iniciado un proceso de disciplina sensible donde muestran comprensión, entendimiento, ponerse en el lugar del niño, lo que implica demostrar empatía por él o ella y darle explicaciones de las consecuencias de sus acciones a través de un método de inducción (Juffer et al., 2008).

A partir de la pregunta sobre una continuidad entre los patrones de cuidado y las relaciones afectivas que se construyen entre padres e hijos y si persisten o no entre varias generaciones de la misma familia y cuáles son los procesos subyacentes, algunos estudios revelan sistemáticamente un efecto de transmisión intergeneracional sustancial 
de los estilos de crianza de los hijos. Según Berlin et al. (2008), se esperaría que los padres que experimentaron un alto grado de responsividad/ sensibilidad en su infancia serían más abiertos a las señales y a las necesidades de sus propios hijos, que aquellos padres que fueron rechazados o tratados de forma ambivalente. Sin embargo, otros estudios han mostrado que los padres pueden reestructurar su representación interna sobre la base de experiencias de apego seguras y significativas después de la infancia (Hesse, 2008). Se podría suponer que algunos de estos padres que, a pesar de no haber tenido en su propia infancia unos padres amorosos y comprensivos, a través de procesos reflexivos personales y experiencias afectivas posteriores, poseen una seguridad lograda (earned security) en su adultez y son capaces de mantener comportamientos sensibles con sus hijos, en condiciones de alto estrés.

En este mismo sentido, se halló que la sensibilidad del cuidador se relaciona con la calidad del desarrollo infantil total, el desarrollo de habilidades de motricidad gruesa y audición y lenguaje en los niños de la muestra. Estos hallazgos confirman la evidencia encontrada en otros estudios que muestran que la calidad de las relaciones tempranas niño-cuidador y la sensibilidad de este último está asociada con el desarrollo de habilidades cognitivas, lingüísticas, sociales, morales y emocionales en los niños (Shonkoff \& Phillips, 2000).

También se encontró que en aquellos padres con menor uso de prácticas disciplinarias moderadas, mayor es la seguridad del apego de sus hijos; mejor es la calidad del cuidado expresado en prontitud, efectividad y consistencia de la respuesta del cuidador, especialmente en términos de la calidad del contacto físico, como forma para la expresión afectiva y emocional con los hijos. Estos resultados son consistentes con la hipótesis sobre la conexión sensibilidad-seguridad que está empíricamente sustentada a través del metaanálisis realizado por De Wolff y van IJzendoorn (1997), que muestra que los cuidadores principales sensibles a las señales y necesidades de los niños, construyen con ellos relaciones de apego seguro, en oposición a aquellos que maltratan y son negligentes en el cuidado, que generan relaciones de apego inseguro (Gómez Muzzio et al., 2008).

Así mismo, se encontró en el estudio que a mayor edad del niño más sensible es el comportamiento del cuidador a sus señales comunicativas y necesidades. Los datos parecen sugerir que en la medida en que el cuidador tiene mayor conocimiento y experiencia interactiva con el niño aprende a responder e interpretar adecuadamente sus comunicaciones y necesidades. Igualmente, se observó una relación inversa entre la edad de la madre y la frecuencia comunicativa verbal. Es decir, a mayor edad materna menor frecuencia comunicativa verbal. La evidencia de 28 países del mundo en vías de desarrollo, con 127000 familias con niños menores de cinco años, muestra que la calidad de la estimulación comunicativa verbal está asociada con el índice de desarrollo humano (HDI) del país (Bornstein \& Putnick, 2012).

Estos hallazgos también podrían estar relacionados con la propia experiencia de infancia de los padres, estresores de la vida cotidiana en la pobreza y con su bajo nivel educativo y de conocimientos respecto a la importancia en la crianza de la estimulación cognitiva, social y emocional con sus hijos, tales como cantar, bailar, jugar, acariciar, contar y leer cuentos y conversar con el niño durante las rutinas de cuidado (Bornstein \& Putnick, 2012; Kim-Cohen et al., 2004).

\section{Conclusiones}

A continuación, se presentan algunos factores protectores y de riesgo de las familias en situación de desplazamiento forzado y reubicación en la ciudad de Bogotá y conjeturas emergentes que se podrían plantear, teniendo en cuenta los análisis anteriores.

\section{Factores protectores}

A partir de los procesos reflexivos realizados por los cuidadores principales sobre sus propias experiencias de cuidado y crianza en la infancia por parte de la generación anterior, se puede hipotetizar que en la mayoría de los cuidadores se ha iniciado un proceso de ruptura con las prácticas de crianza 
transmitidas intergeneracionalmente. Estos procesos reflexivos parecen están encaminados a reducir la intensidad de los patrones de crianza violentos y a buscar estrategias alternativas para establecer normas y límites con sus hijos. Los resultados de las escalas de castigo físico e inversión de roles muestran que algunos cuidadores piensan que existen estrategias alternativas al uso del castigo físico para disciplinar los hijos y otros consideran que es una entre las diversas estrategias para establecer normas y límites, mostrando que en estas familias en condición de desplazamiento, el castigo físico no es la estratégica disciplinaria principal. La mayoría de los cuidadores desarrollan una función parental adecuada y no tienen expectativas de que los hijos pequeños sean la fuente de gratificación de sus necesidades afectivas o instrumentales.

Las asociaciones significativas y positivas encontradas entre la sensibilidad del cuidador y la calidad del desarrollo infantil, especialmente en las áreas de motricidad gruesa y de audición y lenguaje, permiten suponer que los cuidadores principales, cuando son sensibles a las necesidades afectivas y comunicativas de los niños, parecen favorecer un desarrollo infantil adecuado.

\section{Factores de riesgo}

Una minoría de los cuidadores justifica el castigo físico por parte de sus padres en su infancia como una actitud parental de corrección o por su propio bien, lo que llevaría a suponer que los padres con esta percepción respecto al trato recibido por la generación anterior, sería más proclive a repetir el patrón de crianza con estrategias disciplinarias centradas en el castigo físico.

La creencia reportada en el discurso de muchos de los cuidadores es que el castigo físico es el último recurso para disciplinar a los hijos, luego de agotar otras estrategias para el manejo de las normas y límites, sin usar violencia, lo cual podría favorecer la repetición intergeneracional de los patrones de crianza violentos.

Un factor de riesgo respecto al castigo en esta muestra es ser niña, pues se observó una mayor proporción de prácticas disciplinarias severas y moderadas ejercidas sobre las niñas. Sería relevante indagar cuáles son las representaciones de los cuidadores en relación con esta población por género, respecto al comportamiento adecuado y las formas de implementar normas y límites.

Las áreas de desarrollo infantil con menores niveles de desempeño en los niños de la muestra son audición-lenguaje y personal-social. Estas están entre las más afectadas en los niños que viven en contextos de pobreza, debido a diversos factores que actúan sinérgicamente impactando negativamente el desarrollo y aprendizaje infantil, tales como menores niveles educativos maternos, ambientes familiares menos estimulantes cognitiva y socioemocionalmente, mayores niveles de estrés en la vida cotidiana, entre otros (Bornstein \& Putnick, 2012; Wachs, 2009).

\section{Limitaciones del estudio}

Debido a que este es un estudio mixto explicativo con pocos casos, sus resultados son preliminares y no pueden ser generalizados. Por tanto, se requiere hacer un estudio con diseño mixto secuencial explicativo ampliando la muestra de la población en situación de desplazamiento forzado y con reubicación en la ciudad de Bogotá, utilizando un muestreo aleatorizado.

En estudios posteriores es importante evaluar los estado de ánimo del cuidador, especialmente aspectos relacionados con depresión materna, puesto que las madres y padres en contextos de pobreza poseen mayor riesgo de sufrir problemas de salud mental (baja autoestima, creencias negativas sobre su capacidad de control, ansiedad), asociados al estrés fuerte y crónico de condiciones económicas adversas (Shonkoff \& Phillips, 2000; Wachs, 2009) que a su vez afecta la calidad del cuidado infantil (Mulsow, et al., 2002).

Así mismo es relevante evaluar en otros estudios las redes de apoyo familiar, social y comunitario que poseen las familias en situación de desplazamiento, puesto que estas son un factor clave para aminorar el estrés de la crianza, especialmente en condiciones de adversidad psicosocial y afectar la calidad de cuidado (Wachs, 2009) y en consecuencia, la seguridad emocional de los niños/as pequeños. 


\section{Referencias}

Ainsworth, M. D. S., Bell, S. M., \& Stayton, D. F. (1974). Infant-mother attachment and social development: Socialization as a product of reciprocal responsiveness to signals. En M. P. M. Richards (Ed.), The integration of a child in the social world (pp. 99-135). Nueva York: Cambridge University Press.

Ainsworth, M. D. S., Blehar, M. C., Waters, E., \& Wall, S. (1978). Patterns of attachment: A psychological study of the strange situation. Hillsdale, $\mathrm{N} \mathrm{J}$ : Erlbaum.

Álzate, G., Carbonell, O. A., Posada, G., \& Bustamante, M. R. (1999). Escalas cualitativas del comportamiento materno. Manuscrito no publicado, Pontificia Universidad Javeriana, Bogotá, Colombia.

Amnistía Internacional. (16 de julio de 2009) Colombia: Todo queda atrás. Desplazamiento interno en Colombia. Recuperado de http://www.amnesty. org/es/library/info/AMR23/015/2009/es

Arango, M., Acosta, A., \& Jaramillo, M. (2009). Colombia: historia de Marta Arango. En P. Connolly, J. Hayden, D. Levin \& L. Shulman (Eds.), Del conflicto a la construcción de paz: el poder de las iniciativas de la primera infancia (pp. 51-61). Bogotá: Editorial Visuales Dar.

Berlin, L. J. (2007). Interventions to enhance early attachments. The state of the field today. En L. J. Berlin, Y. Ziv, L. Amaya-Jackson \& M. T. Greenberg (Eds.), Enhancing early attachments: Theory, research, intervention, and policy (pp. 3-33). New York: The Guilford Press.

Berlin, L. J., Zeanah, C., \& Lieberman, A. (2008). Prevention and intervention programs for supporting early attachment security. En J. Cassidy \& P. R. Shaver (Eds.), Handbook of attachment: Theory, research, and clinical applications (pp. 745-761). Nueva York: The Guilford Press.

Bornstein, M. C., \& Putnick, D. L. (2012). Cognitive and socioemotional caregiving in developing countries. Child Development, 83, 46-61. doi:10.1111/j.14678624.2011.01673.x

Bowlby, J. (1993). El vínculo afectivo. Barcelona: Editorial Paidós.

Cicchetti, D., Cummings, M.E., Greenberg, M.T., \& Marvin, R.S. (1990). An organizational perspec- tive on attachment beyond infancy. In M.T. Greenberg, D. Cicchetti, \& M.E. Cummings (Eds.), Attachment in the preschool years: Theory, research and intervention (pp-51-86). Chicago: The University of Chicago.

Connolly, P., Hayden, J., Levin, D., \& Shulman, L. (2009). Del conflicto a la construcción de paz: el poder de las iniciativas de la primera infancia. Bogotá: Editorial Visuales Dar.

Consultoría para los Derechos Humanos y el Desplazamiento. (22 de abril de 2009). Victimas emergentes. Desplazamiento, derechos humanos y conflicto armado en 2008 (Boletín informativo № 75). Recuperado de http://www.codhes.org/index. php?option $=$ com_si\&type $=4$

Creswell, J. W., \& Plano Clark, V. (2011). Designing and conducting mixed methods research. Thousand Oaks, CA: Sage.

De Wolff, M. S., \& van IJzendoorn, M. H. (1997). Sensitivity and attachment: A meta-analysis on parental antecedents of infant attachment. Child Development, 68, 571-591. doi:10.2307/1132107

Delage, M. (2010). En el nicho familiar y la superación de las heridas. Barcelona: Gedisa.

Egeland, B., Carlson, E. A., \& Sroufe, L. A. (1993). Resilience as process. Development and Psychopathology, 5, 517-528. doi:10.1017/S0954579400006131

Farah, M. J., Shera, D. M., Savage, J. H., Betancourt, L., Giannetta, J. M., Brodsky, N. L., \& Hurt, H. (2006). Childhood poverty: Specific associations with neurocognitive development. Brain Research, 1110(1), 166-174. doi:10.1016/j.brainres.2006.06.072

Fondo de las Naciones Unidas para la Infancia. (Junio, 2014). Informe anual de UNICEF 2013. Nueva York: Autor. Disponible en http://www.unicef.org/ spanish/publications/index_73682.html

Fondo de las Naciones Unidas para la Infancia. (Mayo, 2006). Hojas informativas sobre la protección de la infancia. Nueva York: Autor. http://www.unicef. org/spanish/protection/files/Hojasinformativas sobre_proteccion_infancia_(Bookl.pdf

Fondo de las Naciones Unidas para la Infancia. (1996). Estado mundial de la infancia [Eejemplar sobre el 50. aniversario]. Nueva York: Autor. Dis- 
ponible en http://www.unicef.org/spanish/sowc/ index_38236.html

Gómez Muzzio, E., Muñoz, M. M., \& Santelices, M. P. (2008). Efectividad de las intervenciones en apego con infancia vulnerada y en riesgo social: un desafío prioritario para Chile. Terapia Psicológica, 26(2), 241-251.

González Viveros, C. (Agosto, 2004). Transformación y resiliencia en familias desplazadas por la violencia hacia Bogotá. Revista de Estudios Sociales, 18, 123 130. Disponible en http://res.uniandes.edu.co/view. php/379/view.php\#*

Hernández Sampieri, R., Fernández Collado, C., \& Baptista Lucio, P. (2010). Metodología de la investigación. México: McGrawHill.

Hesse, E. (2008). The adult attachment interview: Protocol, method of analysis, and empirical studies. En J. Cassidy \& P. R. Shaver (Eds.), Handbook of attachment: Theory, research, and clinical applications (pp. 552-598). Nueva York: The Guilford Press.

Juffer, F., Barkermans-Kranenburg, M. J., \& van IJzendoorn, M. H. (2008). Promoting positive parenting: An attachment-based intervention. Nueva York: Psychology Press.

Kim-Cohen, J., Moffitt T. E., Caspi, A., \& Taylor, A. (2004). Generic and environmental processes in young children's resilience and vulnerability to socioeconomic deprivation. Child Development, 75, 651-668. doi: 10.1111/j.1467-8624.2004.00699.x

Mulsow, M., Caldera, Y., Pursley, M., Reifman, A., \& Huston, A. (2002). Multilevel factors influencing maternal stress during the first three years. Journal of Marriage and Family, 64, 944-956. doi:10.1111/ j.1741-3737.2002.00944.x

Myers-Walls, J. A., Myers-Bowman, K. S., \& Posada, G. (2005). Parenting practices worldwide. En B. Ingoldsby \& S. Smith (Eds.), Families in global and multicultural perspective (pp. 147-167). Newbury Park: Sage.

Ortiz, N. (1991). Escala Abreviada de Desarrollo. Bogotá: Ministerio de Salud.

Pederson, D. R., Gleason, K. E., Moran, G., \& Bento, S. (1998). Maternal attachment representations, maternal sensitivity, and the infant-mother attachment relationship. Developmental Psychology, 34, 925-933. doi:10.1037/0012-1649.34.5.925
Pederson, D. R., \& Moran, G. (1995). Appendix B. Maternal Behavior Q-set. En E. Waters, B. E. Vaughn, G. Posada \& K. Kondo-Ikemura (Eds.), Caregiving, cultural, and cognitive perspectives on secure base behavior and working models: New growing points of attachment theory and research. Monographs of the Society for Research in Child Development, 60(23 Serial N. 244), 247-254. doi:10.2307/1166182

Posada, G., Carbonell, O. A., Alzate, G., \& Plata, S. J. (2004). Through Colombian lenses: Ethnographic and conventional analyses of maternal care and their associations with secure base behavior. Developmental Psychology, 40, 508-518. doi:10.1037/0012-1649.40.4.508

Posada, G., Jacobs, A., \& Richmond, M. (1998). The maternal behavior for preschoolers Q-set. Unpublished manuscript. Purdue University at West Lafayette, IN.

Posada, G., Kaloustian, G., Richmond, M.K., \& Moreno, A. J. (2007). Maternal secure base support and preschoolers' secure base behavior in natural environments. Attachment \& Human Development, 9, 1-19.

Posada, G., \& Waters, E. (1990). Family Behavior Survey. Part IV. Manuscrito inédito, Psychology Department, Stony Brook, New York University, NuevaYork, Estados Unidos.

Sagi-Schwartz, A. (2012). Children of war and peace: A human development perspective. Journal of Conflict Resolution, 56(5), 933-951. doi:10.1177/00222712446128

Shonkoff, J. P., \& Phillips, D. A. (Eds.). (2000). From neurons to neighborhoods. The science or early childhood development. Washington, DC: National Academic Press. Disponible en http://www.nap. edu/openbook.php?record_id $=9824$

Solís-Cámara, P., \& Bavolek, S. (1995). Manual del Inventario de Paternidad para adultos y adolescentes (MIPPA). Park City, UT: Family Development Resources Inc.

Solís-Cámara, P., \& Díaz Romero, M. (2006). Efectos de un programa de crianza para mamás y papás de niños pequeños: la importancia del nivel educativo de los padres. Revista Infancia, Adolescencia y Familia, 1(1), 161-176. 
Strauss, A., \& Corbin, J. (2002). Bases de la investigación cualitativa: técnicas y procedimientos para desarrollar la teoría fundamentada. Medellín: Universidad de Antioquia.

Thompson Gershoff, E. (2002). Corporal punishment by parents and associated child behaviors and experiences: A meta-analytic and theoretical review. Psychological Bulletin, 128, 539-579. doi: 10.1037//0033-2909.128.4.539

Uribe-Mallarino, C. (2008). Estratificación social en Bogotá: de la política pública a la dinámica de la segregación social. Universitas Humanística, 65, $139-171$

Velasco Bernal, V. L., \& Lozano Mancera, N. (2012). Comportamiento de la violencia intrafamiliar, Colombia, 2012. Bogotá: Instituto Nacional de Medicina Legal y Ciencias Forenses.

Wachs, T. D. (julio, 2009). La naturaleza y las consecuencias del estrés sobre las familias que viven en países con bajos ingresos. En El estrés familiar: protegiendo el bienestar de los más pequeños (pp. 3-10). La Haya: Bernard van Leer Foundation. Recuperado de file://C:/Users/HP/Desktop/

El_estres_familiar_Protegiendo_el_bienestar_ de los mas pequenos.pdf

Waters, E. (1995). The attachment Q-set (version 3). En E. Waters, B. Vaughn, G. Posada, \& K. KondoIkemura (Eds.), Caregiving, cultural, and cognitive perspectives on secure-base behavior and working models: New growing points of attachment theory and research (pp. 234-246). Monographs of the Society for Research in Child Development, 60(2-3, Serial N.․ 244). doi:10.2307/1166181

Wyman, P., Cowen, E. L., Work, W. C., Hoyt-Meyers, L., Magnus, K., \& Fagen, D. B. (1999). Caregiving and developmental factors differentiating young atrisk urban children showing resilient versus stressaffected outcomes: A replication and extension. Child Development, 70, 645-659. doi:10.1111/14678624.00047 